\title{
THERMOREVERSIBLE IN-SITU NASAL GEL FORMULATIONS AND THEIR PHARMACEUTICAL EVALUATION FOR THE TREATMENT OF ALLERGIC RHINITIS CONTAINING EXTRACTS OF MORINGA OLIFERA AND EMBELIA RIBES
}

\author{
RICHA SRIVASTAVA', SAJAL SRIVASTAVA ${ }^{2}$, SATYA PRAKASH SINGH ${ }^{3}$
}

${ }^{1}$ Amity Institute of Pharmacy, Amity University Gomti Nagar Extention, Lucknow UP, 226028 India, ${ }^{2}$ Deputy Director, Amity Institute of Pharmacy, Amity University, Gomti Nagar Extention, Lucknow UP, 226028 India, ${ }^{3}$ Pharmacy Department Integral University, Lucknow UP 226026 India

Email: richa0306@rediffmail.com

Received: 30 Mar 2017, Revised and Accepted: 10 Oct 2017

\begin{abstract}
Objective: The present study was planned to develop thermo-reversible in-situ gel for the treatment of allergic rhinitis (AR). The objective of the present investigation was to develop a mucoadhesive in-situ gel with reduced nasal mucocilliary clearance to improve the local effect of the polyherbal extract in the treatment of allergic rhinitis (AR). The prolonged residence of drug formulation in the nasal cavity is one of utmost importance for intranasal drug delivery. The prepared formulations were subjected for gelling temperature, gelling time, viscosity, gel strength, $\mathrm{pH}$, drug content, mucoadhesive strength, spread ability and irritancy studies.
\end{abstract}

Methods: In the study the pluronic F127 (PF127) based mucoadhesive in-situ nasal gels containing Moringa olifera (MO) and Embelia ribes (ER) extracts were used having antioxidant and anti-inflammatory effect. A polyherbal thermosensitive in-situ hydrogel was designed and evaluated by the mixing of pluronic F127, poly (ethylene glycol) (PEG400) and Xanthan gum with a small amount of (hydroxypropyl methylcellulose) HPMC K4M and Carbopol 934. Total 13 thermosensitive in-situ gels of extracts were prepared through combination of HPMC K4M or Carbopol or xanthan gum and PF127. All the preparations were investigated, and the selected method for gel formation underwent the thermal transition from sol to hydrogel.

Results: The mucoadhesive gel after being administered into the nasal cavity, get transformed into the viscous hydrogel at body temperature, which diminished nasal mucociliary clearance and prolonged the duration of action. The in-situ nasal herbal gel prepared by combination of different concentration of to HPMC K4M or carbopol or xanthan gum with PF127 (10\% w/v) produces the better and effective gel. The findings of evaluation parameter indicate that the in-situ gel prepared by combination with corbopol were better quality compared to HPMC K4M and xanthan gum.

Conclusion: From these findings, it can be concluded that in-situ herbal nasal gels may be potential drug delivery systems for Moringa olifera and Embelia ribes extracts to overcome first-pass metabolism and thereby to improve the bioavailability. The mucoadhesive in-situ gel system is a promising approach for the intranasal delivery of polyherbal extracts for the therapeutic effects improvement of Allergic rhinitis.

Keywords: Moringa olifera, Embelia ribes, HPMC, Carbopol, In-situ, Pluronic F127

(C) 2017 The Authors. Published by Innovare Academic Sciences Pvt Ltd. This is an open access article under the CC BY license (http://creativecommons.org/licenses/by/4.0/) DOI: http://dx.doi.org/10.22159/ijap.2017v9i6.18780

\section{INTRODUCTION}

Allergic rhinitis (AR) is a heterogeneous disorder, which is often characterized by mucosal infiltration and actions of eosinophils, plasma cells, and mast cells. This disorder is extremely widespread but its diagnosis and prevention is very poor. Presently, various types of drug formulations for Allergic rhinitis are available. The practices of nasal formulation are limited due to associate constraints with drug delivery systems poses a major drawback. The factors affecting the drug delivery system comprise volume of the nasal cavity for the drug volume $(<0.2 \mathrm{ml})$, mucociliary clearance, and anterior discharge [1-3].

Generally synthetic antihistamines are used to provide symptomatic relief to allergic symptoms due to histamine release. The synthetic drugs are associated with various types of side effects [4]. Herbal medicines have been widely used all over the world since ancient times and have been recognized by physicians and patients for their better therapeutic value as they have fewer adverse effects as compared with modern medicines. Natural molecules derived from plant extracts offer a particularly exciting avenue for further research. Plant extracts, however, are often ill-defined as to the method of extraction, plant-to-solvent ratio and the content of active ingredients. Moreover, the stability of the colour, odour, transparency and/or active ingredients with time is also often a limiting factor $[5,6]$. Plant extracts are different in several respects from purified therapeutic agents. Firstly, they are more dilute than the pure chemicals that are familiar to us; secondly herbs often contain additional active principles that may be closely related both chemically and therapeutically to the constituent primarily responsible for its effects. Phytotherapeutics need a scientific approach to deliver the components in a sustained manner to increase patient compliance and avoid repeated administration [7].

In recent years, the novel in-situ gelling formulations are progressing rapidly especially in the field of nasal drug delivery. There is an increase in the number of in-situ forming systems has been formulated and developed for the various biomedical parameters out of the different preparations the thermally induced gelling systems had to be the most challenging one, for the development of nasal drug delivery systems. The nasal mucosa has seriously emerged as a therapeutically viable route for the systemic drug delivery. In addition, intranasal absorption avoids the gastrointestinal and hepatic presystemic metabolism, enhancing drug [8].

Among the different nasal drug delivery systems, in-situ gel formulations have been explored for both local and systemic drug delivery. These drug delivery systems exist in sol form before their administration; however, once administered, they undergo gelation to form a gel. The factors regulating the in-situ gel formation process include microenvironment temperature, changes in $\mathrm{pH}$, presence of ions, ultraviolet irradiation, and polymers. Rheological properties of gels, which are critical to their efficacy, are important in retaining the gel at the site of application or absorption [9]. The plant used in formulation was selected based on scientifically reported property. In the present study we have selected Moringa olifera (MO) and Embelia ribes (ER) for the formulation of herbal nasal gel. Therefore, 
an attempt has been made to formulate pluronic F127 (PF127) based mucoadhesive in-situ nasal gels containing Moringa olifera and Embelia ribes to enhance its therapeutic effect.

\section{MATERIALS AND METHODS}

\section{Materials}

Moringa olifera fresh leaves were collected and dried; Embelia ribes fruit (dreid) were brought from local market. The leaves and seeds were authenticated by State Ayurvedic College Lucknow with specimen no. 17 and 19 respectively. Pluronic F127 (PF127) was supplied from Sigma Aldrich Pvt. Ltd India, hydroxypropyl methylcellulose K4M, Xanthan gum, and Carbopol 934 were from SDFCL Pvt Ltd. India. All the other chemicals and reagents used in this study were of analytical grade.

\section{Methods}

\section{Preparation of extracts}

Air dried and coarsely powdered (500 gm) of Moringa olifera leaves and Embelia ribes fruits, were weighed and their hydroalcoholic extracts were prepared separately, by using distilled water and ethanol. The extracts were then concentrated to dryness under reduced pressure and controlled temperature, respectively and they were preserved in a refrigerator.

\section{Preparation of thermorevresible in-situ nasal polyherbal gel by cold method}

The thermorevresible nasal in-situ gel formulation was prepared by cold method [10]. For preparation of PF127 solutions, the required amount of polymer was dispersed in distilled, deionized water with continuous stirring for $1 \mathrm{~h}$. The partially dissolved pluronic solutions were stored in the refrigerator until the polymer was completely dissolved (approximately $24 \mathrm{~h}$ ). The preparation of hydroxypropyl methylcellulose K4M solution is the same as that of PF127. The carbopol 934/pluronic F127 mixed solutions, hydroxypropyl methylcellulose K4M/PF127 and xanthan gum/PF127 mixed solutions were prepared by dispersing the required amount of PF127in the desired concentration of carbopol 934 or HPMC K4M with continuous stirring for $1 \mathrm{~h}$, respectively. Different isotonicity agents, benzalkonium chloride (Fisher Scientific. India.) (as preservative) and the desired amount of Moringa olifera $(2 \% \mathrm{w} / \mathrm{w})$ and Embelia ribes $(2 \% \mathrm{w} / \mathrm{w})$ were added in the solution. The samples were then allowed to equilibrate at $4{ }^{\circ} \mathrm{C}$ overnight $(24 \mathrm{~h}$ at least) [11]. The composition of nasal gel is given in (table 1).

Table 1: Composition of different thermoreversible in-situ nasal polyherbal gels

\begin{tabular}{|c|c|c|c|c|c|c|c|c|c|c|c|c|c|}
\hline \multirow[t]{2}{*}{ Ingredients } & \multicolumn{13}{|c|}{ Formulations } \\
\hline & F1 & F2 & F3 & F4 & F5 & F6 & F7 & F8 & F9 & F10 & F11 & F12 & F13 \\
\hline $\mathrm{MO}(\% \mathrm{w} / \mathrm{v})$ & 2 & 2 & 2 & 2 & 2 & 2 & 2 & 2 & 2 & 2 & 2 & 2 & 2 \\
\hline $\mathrm{ER}(\% \mathrm{w} / \mathrm{v})$ & 2 & 2 & 2 & 2 & 2 & 2 & 2 & 2 & 2 & 2 & 2 & 2 & 2 \\
\hline Pluronic F127 (\%w/v) & 16 & 14 & 12 & 10 & 10 & 10 & 10 & 10 & 10 & 10 & 10 & 10 & 10 \\
\hline Carbopol $934(\% \mathrm{w} / \mathrm{v})$ & - & - & - & - & 0.5 & 1.0 & 1.5 & - & - & - & - & - & - \\
\hline Hydroxypropyl methylcellulose K4M(\%w/v) & - & - & - & - & - & - & - & 0.5 & 1.0 & 1.5 & - & - & - \\
\hline Xanthan gum $(\% \mathrm{w} / \mathrm{v})$ & - & - & - & - & - & - & - & - & - & - & 0.5 & 1.0 & 1.5 \\
\hline Benzalkonium chloride $(\% \mathrm{v} / \mathrm{v})$ & 0.01 & 0.01 & 0.01 & 0.01 & 0.01 & 0.01 & 0.01 & 0.01 & 0.01 & 0.01 & 0.01 & 0.01 & 0.01 \\
\hline Distilled water (ml) & q. s. & qs & qs & qs & qs & qs & qs & qs & qs & qs & qs & qs & qs \\
\hline
\end{tabular}

MO: Moringa olifera extract \%w/v, ER: Embelia ribes extract \%w/v

\section{Evaluation of thermoreversible in-situ nasal polyherbal gels}

\section{Gelling temperature and gelling time}

Gelling temperature refers to the temperature when the meniscus of the formulation would no longer move upon slanting the test tubes at $90^{\circ}$ angle. The gelling temperature was determined by placing the test tube, containing sufficient quantity of the prepared solutions, in a water bath at $4{ }^{\circ} \mathrm{C}$. The temperature of water bath was increased slowly at a constant rate of $1^{\circ} \mathrm{C}$ in every $2 \mathrm{~min}$.

Gelling time was recorded as the time for first detection of gelation. The sol-gel transition temperature (Tsol-gel) of the prepared insitu gel formulations was evaluated by transferring $2 \mathrm{ml}$ of the prepared formulation to a test tube $(10 \mathrm{ml})$, with a diameter of $1.0 \mathrm{~cm}$. After sealing with a parafilm, the tube was kept in a circulation water bath at $37^{\circ} \mathrm{C}$. Following each temperature setting, equilibration was allowed for $10 \mathrm{~min}$. Finally, the test tube was placed horizontally to observe the state of the sample and to examine the gelation. All the measurements were performed in triplicates.

\section{Viscosity of solution}

Viscosity of the in-situ gel systems was determined using Brookfield viscometer coupled with S-94 spindle (Brookfield Engineering Laboratories Inc., MA, USA). The prepared gel formulations were transferred to the beaker. The spindle was lowered perpendicularly into the gel at $100 \mathrm{rpm}$ and temperature was maintained at $37 \pm 0.5$ ${ }^{\circ} \mathrm{C}$. The viscosity was determined during the cooling of the system. All the measurements were performed in triplicates.

\section{Determination of $\mathrm{pH}$}

One $\mathrm{ml}$ of the prepared gels was transferred to a $10 \mathrm{ml}$ volumetric flask, and the solution was diluted with distilled water. The $\mathrm{pH}$ of resulting solution was determined using a digital $\mathrm{pH}$ meter (Elico
LI120, India), which was previously calibrated using phosphate buffers at $\mathrm{pH} 4$ and $\mathrm{pH} 7$.

\section{Drug content assay}

One $\mathrm{ml}$ of the prepared formulation was dispersed in $10 \mathrm{ml}$ of methanol for 2-3 min with occasional shaking. The resulting solution was filtered through a $0.45 \mu \mathrm{m}$ filter paper and was diluted with methanol. The amount of flavonoids present in the formulation was determined spectrophotometrically at $275 \mathrm{~nm}$ (Shimadzu UV1800, Japan).

\section{Gel strength}

\section{Mucoadhesive strength}

Ex vivo mucoadhesive strength was determined using fresh sheep nasal mucosa. The mucosal membrane was separated by removing the underlying fat and loose tissues. The membrane was washed thrice with distilled water and phosphate buffer (pH 6.4). Modified balance method was used to design the experiment.

The balance was equilibrated on both sides by placing one beaker on the left pan and a weight ( $5 \mathrm{~g}$ ) on the opposite pan. The sheep nasal mucosa was cleaved into $1 \mathrm{~cm}^{2}$ and glued with cyanoacrylate over the glass support to allow the smooth surface of nasal mucosa face the upper side of the glass. The glued sheep nasal mucosa was wetted with phosphate buffer ( $\mathrm{pH}$ 6.4) by filling the beaker with the buffer on the right-hand side of the balance by lowering the glass support. The above setup was placed under the right side of the pan.

A thin film of the prepared gel $(1 \mathrm{~g})$ was spread on the lower surface of the right pan. The right pan was lowered and was spread with gel by removing the beaker from the left pan. The pan was left undisturbed for 2 min to ensure proper contact between the nasal mucosa and the gel. Following this, water was slowly added to the left pan using a burette until the nasal mucosa was separated from 
the gel film. The mucoadhesive force was calculated by determining the weight required to separate the mucosa. The force was expressed in dynes per square centimeter $\left(\right.$ dyne $\left./ \mathrm{cm}^{2}\right)$.

\section{Spreadability}

Spreadability was determined using a $10 \times 4 \mathrm{~cm}$ rectangular glass slide. The sheep nasal mucosa from serosal side was tied on the surface of slide with a thread. The slide was kept in a hot air oven, at $37{ }^{\circ} \mathrm{C}$ and one drop of gel was placed on the mucosa at an angle of $120^{\circ}$. Spreadability was determined relative to the distance travelled by the drop of gel (liquid) before its gelation. Average of three readings was recorded [12-16].

\section{Animals}

Healthy swiss mice $(20-25 \mathrm{~g})$, were obtained from the Centarl drug research institute (Lucknow, India). Prior to experimentation, the animals were quarantine for at least one week to a 12-h light/dark cycle All experimental protocols were from the CPCSEA (AUUP/AIP/ $\mathrm{PhD} / 006 / 2017$ ) and approved by Institutional Animal Ethics Committee.

\section{Irritancy study}

Mark an area $\left(5 \mathrm{~cm}^{2}\right)$ on the left hand dorsal surface. The cream was applied to the specified area and time was noted. Irritancy, erythema, edema, was checked if any for regular intervals up to $24 \mathrm{~h}$ and reported.

It is done by using swiss mice. The back skin of area of $5 \mathrm{~cm}^{2}$ is used. If the formulation produced scores of 2 or less they are considered to have no skin irritation $[5,6]$.
- Erythemia scale: none-0, slight-1, well defined-2, moderate-3, scar formulation-4.

- Edema scale: none-0, slight-1, well defined-2, moderate-3, severe-4.

\section{RESULTS AND DISCUSSION}

Gelling temperature, gelling time and viscosity of in-situ nasal herbal gels

The thirteen different in-situ nasal herbal gels were formulated using HPMC, carbopol and xanthan gum as the base polymers. The results of gelling temperature, gelling time and viscosity of herbal gels are demonstrated in (table 2).

The gelling temperature of the in-situ nasal herbal gel ranged between $26.14{ }^{\circ} \mathrm{C}$ and $36.17{ }^{\circ} \mathrm{C}$. The findings of the gelling temperature of prepared gel indicate that suitable for thermo reversible nasal gel.

The gelling time of herbal gel ranged between 5.2 and $11.3 \mathrm{sec}$ (table 2). The gelling temperature of formulation was found to be within the range. The gelling time increases on decreasing the concentration of F4.

The viscosity of the in-situ nasal herbal gels ranged between 30.2 and $40.3 \mathrm{~Pa} \cdot \mathrm{s}$. The viscosity of in-situ nasal herbal gels enhance on higher concentration of PF127. Similarly, on increasing the concentration of HPMC K4M/carbopol/xanthan gum, it enhances the viscosity of formulation.

Table 2: Gelling temperature, gelling time and viscosity of solution thermo reversible in-situ nasal polyherbal gel formulations

\begin{tabular}{llll}
\hline Formulation & Gelation temperature $\left({ }^{\circ} \mathbf{C}\right)$ & Gelling time (sec) & Viscosity/(Pa·s) \\
\hline F1 & $26.14 \pm 0.25$ & $5.2 \pm 0.05$ & $40.3 \pm 0.82$ \\
F2 & $29.35 \pm 0.47$ & $7.5 \pm 0.08$ & $35.7 \pm 0.15$ \\
F3 & $32.52 \pm 0.63$ & $8.1 \pm 0.02$ & $32.3 \pm 0.37$ \\
F4 & $36.17 \pm 0.43$ & $11.3 \pm 0.6$ & $29.5 \pm 0.64$ \\
F5 & $31.24 \pm 0.81$ & $9.1 \pm 0.07$ & $32.2 \pm 0.84$ \\
F6 & $30.83 \pm 0.51$ & $8.5 \pm 0.02$ & $32.4 \pm 0.39$ \\
F7 & $29.45 \pm 0.64$ & $6.2 \pm 0.04$ & $32.9 \pm 0.67$ \\
F8 & $32.18 \pm 0.38$ & $10.5 \pm 0.14$ & $31.5 \pm 0.53$ \\
F9 & $31.38 \pm 0.43$ & $9.3 \pm 0.09$ & $31.8 \pm 0.45$ \\
F10 & $31.02 \pm 0.37$ & $7.6 \pm 0.11$ & $32.1 \pm 0.68$ \\
F11 & $33.41 \pm 0.95$ & $10.8 \pm 0.04$ & $30.2 \pm 0.47$ \\
F12 & $32.52 \pm 0.17$ & $9.7 \pm 0.17$ & $30.4 \pm 0.92$ \\
F13 & $31.61 \pm 0.58$ & $8.5 \pm 0.13$ & $30.5 \pm 0.48$ \\
\hline
\end{tabular}

Values are mean \pm SD of three determinations

\section{Gel strength, ph and drug content of in-situ nasal herbal gel}

The outcomes of gel strength, $\mathrm{pH}$ and drug content of in-situ nasal herbal gel are displayed in (table 3). The gel strength of in-situ nasal herbal gel was ranged between 53.1 to $63.7 \mathrm{~s}$. It was observed on higher concentration of Carbopol 934 exhibited maximum gel strength. The addition of HPMC K4M/Carbopol/Xanthan gum in PF127 resulted to increase the gel strength.

Gel pH was in the range of 5.2 to 5.9 which was in the range of $\mathrm{pH}$ at the absorption site (4.5-6.5). The drug content of the in-situ nasal herbal gels ranged between $90.4 \%$ and $93.4 \%$.

Table 3: Gel strength, $\mathrm{pH}$ and drug content of thermo reversible in-situ nasal polyherbal gel formulations

\begin{tabular}{llll}
\hline Formulation & pH & Gel strength (s) & Drug content \\
\hline F1 & $5.2 \pm 0.05$ & $62.1 \pm 0.14$ & $91.5 \pm 0.68$ \\
F2 & $5.2 \pm 0.01$ & $57.5 \pm 0.09$ & $92.7 \pm 0.49$ \\
F3 & $5.4 \pm 0.07$ & $55.8 \pm 0.28$ & $90.4 \pm 0.24$ \\
F4 & $5.6 \pm 0.02$ & $53.1 \pm 0.04$ & $93.4 \pm 0.63$ \\
F5 & $5.4 \pm 0.09$ & $55.4 \pm 0.42$ & $91.8 \pm 0.82$ \\
F6 & $5.5 \pm 0.05$ & $58.3 \pm 0.63$ & $92.6 \pm 0.67$ \\
F7 & $5.7 \pm 0.04$ & $63.7 \pm 0.18$ & $92.5 \pm 0.43$ \\
F8 & $5.5 \pm 0.08$ & $54.5 \pm 0.34$ & $91.7 \pm 0.54$ \\
F9 & $5.8 \pm 0.03$ & $56.2 \pm 0.61$ & $92.3 \pm 0.28$ \\
F10 & $5.9 \pm 0.02$ & $59.6 \pm 0.58$ & $90.7 \pm 0.17$ \\
F11 & $5.4 \pm 0.09$ & $54.1 \pm 0.47$ & $92.3 \pm 0.34$ \\
F12 & $5.6 \pm 0.07$ & $55.8 \pm 0.34$ & $93.1 \pm 0.49$ \\
F13 & $5.8 \pm 0.02$ & $57.2 \pm 0.19$ & $91.6 \pm 0.26$ \\
\hline
\end{tabular}

Values are mean \pm SD of three determinations 


\section{Mucoadhesive strength and spreadability of in-situ nasal herbal gel}

The findings of mucoadhesive strength and spreadability of in-situ nasal herbal gel are exhibited (table 4). The mucoadhesive strength was ranged between 6317.2 to $4236.7 \pm 0.61 \mathrm{dyne} / \mathrm{cm}^{2}$. The higher concentration of Carbopol 934 produced greater mucoadhesive strength. Mucoadhesive drug delivery systems allows rapid dissipation of drug in the circulatory system, thereby preventing the first-pass metabolism and prolonging the residence time of the dosage at the site of application or absorption. In the present study, the formulations prepared with high concentration of HPMC/ carbopol/xanthan gum exhibited more mucoadhesion strength as compared to PF127 (10\%). The spreadability of in-situ nasal herbal gel ranged between $7.6 \pm 0.21$ to $11.7 \pm 0.65 \mathrm{~cm}$.

Table 4: Mucoadhesive strength and spreadability of thermoreversible in-situ nasal polyherbal gel formulations

\begin{tabular}{|c|c|c|}
\hline Formulation & Mucoadhesive strength (dyne $/ \mathrm{cm}^{2}$ ) & Spreadability (cm) \\
\hline F1 & $6154.2 \pm 0.53$ & $7.6 \pm 0.21$ \\
\hline F2 & $5236.8 \pm 0.29$ & $9.2 \pm 0.53$ \\
\hline F3 & $4914.5 \pm 0.73$ & $10.8 \pm 0.43$ \\
\hline F4 & $4236.7 \pm 0.61$ & $12.3 \pm 0.17$ \\
\hline F5 & $4862.9 \pm 0.38$ & $11.7 \pm 0.65$ \\
\hline F6 & $5543.4 \pm 0.92$ & $11.1 \pm 0.34$ \\
\hline F7 & $6317.2 \pm 0.68$ & $10.2 \pm 0.41$ \\
\hline F8 & $4567.5 \pm 0.51$ & $10.7 \pm 0.57$ \\
\hline F9 & $5247.9 \pm 0.36$ & $9.3 \pm 0.83$ \\
\hline F10 & $6153.3 \pm 0.18$ & $8.8 \pm 0.19$ \\
\hline F11 & $4365.7 \pm 0.54$ & $11.2 \pm 0.32$ \\
\hline F12 & $4739.2 \pm 0.32$ & $10.5 \pm 0.48$ \\
\hline F13 & $5934.8 \pm 0.47$ & $9.1 \pm 0.56$ \\
\hline
\end{tabular}

Values are mean $\pm \mathrm{SD}$ of three determinations

\section{Adverse effect of in-situ nasal herbal gel}

The in-situ nasal herbal gel show no redness, edema, inflammation and irritation during irritancy studies (table 5). The F3, F4, F6, F7,
F12 and F13 produced slight irritation, edema and erythema, but it is acceptable.

The prepared in-situ nasal herbal gels were safe to use.

Table 5: Adverse effect thermo reversible in-situ nasal polyherbal gel formulations

\begin{tabular}{llll}
\hline Formulations & Irritant & Erythema & Edema \\
\hline F1 & 0 & 0 & 0 \\
F2 & 0 & 0 & 0 \\
F3 & 0 & 1 & 0 \\
F4 & 1 & 0 & 0 \\
F5 & 0 & 0 & 0 \\
F6 & 0 & 1 & 0 \\
F7 & 1 & 0 & 1 \\
F8 & 0 & 0 & 0 \\
F9 & 0 & 0 & 0 \\
F10 & 0 & 0 & 0 \\
F11 & 0 & 0 & \\
F12 & 1 & 0 & 0 \\
F13 & 0 & 0 & \\
& & 0 \\
\end{tabular}

In the past few years, an increasing number of in-situ gel forming systems have been reported for various biomedical applications, including drug delivery, cell encapsulation, and tissue repair. These were in the form of solutions, but gelation could occur in-situ by ionic cross-linking or a change of ph or temperature. The latter approach is based on temperature induced phase transition. The pluronics are made from ABA-type triblock copolymers containing PEO (A) and PPO units (B). Further the concentrated aqueous solutions of pluronic form thermo reversible gels.

The gel containing pluronic produces weak mechanical strength, rapid erosion, and the non-biodegradability of PEO-PPO-PEO, which prevented the use of high molecular weight polymers. Hence, to minimize the associated drawback, it is required to formulate the pluronic with other bioadhesive polymers namely carboxymethylcellulose (CMC), hydroxypropylmethyl cellulose (HPMC K4M), carbopol, xanthan gum, methyl methacrylate etc. We planned to restrain the viscoelastic properties and the bioadhesive characteristics of PF127 by forming dual systems with a high molecular weight HPMC K4M/Carbopol/xanthan gum $[17,18]$. The object of the present study to formulate in-situ nasal herbal gel, modulating the viscoelastic properties and the bioadhesive characteristics of PF127by forming dual systems with a high molecular weight HPMC K4M or carbopol.

Formulation of in-situ gels appears very attractive since it is fluid like prior to nasal administration and can thus easily be instilled as a drop allowing accurate dosing, but sets into a gel with increased residence time at body temperature.

PF127 has excellent thermo sensitive gelling properties, low toxicity and irritation, excellent water solubility, good release characteristics and compatibility with other excipients. HPMC K4M/carbopol/ xanthan gum was selected as mucoadhesive agent. PF127 is more soluble in cold water than in hot water therefore gels were prepared by cold technique.

Table 2 demonstrated with the increase of concentration of HPMC $\mathrm{K} 4 \mathrm{M}$ or carbopol or xanthan gum, temperature was lessened and viscosity was raised moderately. Thus, packing of micelles and micelle entanglements might be the possible mechanisms of pluronic solution gelation with increase of temperature. PF127 solution behaves as a mobile viscous liquid at room temperature $\left(25^{\circ} \mathrm{C}\right)$, and transformed into a semi-solid transparent gel at body temperature $\left(37^{\circ} \mathrm{C}\right)$. Pluronic formulations generally increase drug residence time at application 
sites through gelling, resulting in improved bioavailability and efficacy. Block copolymer PF127gels were thought to be formed by hydrogen bonding in aqueous systems, caused by the attraction of the pluronic ether oxygen atom of the ethyleneoxide chain with protons of the carbopol carboxylic groups or hydroxypropylmethyl cellulose hydroxyl groups, which contributed to the aggregation [17]. The desired gel strength may be achieved by combination of 2 polymers, so, PF127 concentration could be reduced to lower toxicity of huge PF127content.

The experiments about effect of combination of bioadhesive agents and PF127 on gelation temperature and rheology behavior demonstrated that the packing and entanglements of micelles were promoted by adding either HPMC K4M or carbopol or xanthan gum, which accordingly lead to decrease of gelation temperature. There was some optimization about gelation temperature to ensure a solution at room temperature $\left(25^{\circ} \mathrm{C}\right)$, and a semi-solid transparent gel at body temperature $\left(37^{\circ} \mathrm{C}\right)$ [17]. These results suggested that the optimum concentration for HPMC K4M or carbopol or xanthan gum solution used in-situ gel forming system was $0.5 \%-1.5 \%(\mathrm{w} / \mathrm{v})$, and that of pluronic F127 was below $10 \%(\mathrm{w} / \mathrm{v})$.

The viscosity of the formulated herbal gels was increased with the increment in the amount of hydroxypropylmethyl cellulose HPMC K4M or carbopol or xanthan gum. The viscosity was directly dependent on the bioadhesive content of the formulations. The hydroxypropylmethyl cellulose HPMC K4M or carbopol or xanthan gum can markedly prolong the residence time of the drugs in the nasal cavity because of their desirable mucoadhesive property. This can facilitate the sustained release of the drug can be maintained due to the high viscosity of the cellulose following hydration in the nasal cavity. Consequently, it increases the intranasal bioavailability of both small hydrophobic and hydrophilic macromolecular drugs $[10,19]$.

The presence of combination of HPMC K4M or carbopol or xanthan gum significantly increased the viscosity as well as gel strength. In addition, the formulations prepared with high concentration of HPMC K4M were associated less spread ability. This was attributed to the high viscous nature of HPMC K4M. The results of ph of the formulations did not show any mucosal irritation because all the formulations ph was within in the acceptable range. Formulation should possess mild acidic ph for activation of lysozyme (A natural antibacterial enzyme important for controlling nasal microbial count which becomes inactive at alkaline $\mathrm{pH}$ ) [13].

Mucoadhesion involves 3 stages: wetting; interpenetration; and mechanical interlocking between mucin and polymer. Owing to the characteristic anatomy and physiology of the nasal passage, i.e., large surface area, highly vascularized epithelium, porous endothelial membrane, nasal drug delivery has emerged as a promising route of drug administration for the systemic therapy. The combination of HPMC K4M or carbopol or xanthan gum increases the mucoadhesive strength resulting to enhance the retention of drug in endothelial membrane [20]. The in-situ nasal herbal gel show no redness, edema, inflammation and irritation during irritancy studies, and it was safe to use. The in-situ nasal herbal gel prepared by combination of different concentration of to HPMC K4M or carbopol or xanthan gum with PF127 (10\% w/v) produces the better and effective gel. The findings of evaluation parameter indicate that the in-situ gel prepared by combination with corbopol were better quality compared to HPMC K4M and xanthan gum.

\section{CONCLUSION}

The outcomes of the present study indicate that extract of Moringa olifera and Embelia ribes was successfully incorporated into the formulation to obtain in-situ nasal gel. The formulated in-situ nasal herbal gel showed good gelling temperature, gelling time, viscosity, gel strength, ph, drug content, mucoadhesive strength and spreadability. The novel mucoadhesive in-situ gels containing plant extract was developed to overcome the first-pass metabolism and enhance the subsequent low bioavailability of the drug. However, future in vivo studies are required to confirm these results.

\section{CONFLICT OF INTERESTS}

The authors declare no conflict of interests

\section{REFERENCES}

1. Togias AG. Systemic immunologic and inflammatory aspects of allergic rhinitis. J Allergy Clin Immunol 2000;106:S247-S250.

2. Skoner DP. Allergic rhinitis: definition, epidemiology, pathophysiology, detection, and diagnosis. J Allergy Clin Immunol 2001;108:S2-S8.

3. Capkova Z, Vitkova Z, Subova M. Formulation of loratadine into hydrogels. Acta Fac Pharm Univ Comenianae 2005;52:73-8.

4. Kisan RJ, Manoj NG, Ishaque MS, Vilarsrao JK, Sambjahi SP. Nasal drug delivery system-factors affecting and applicatins. Curr Drug Ther 2007;2:27-38.

5. Basu S, Bandyopadhyay AK. Development and characterization of mucoadhesive in-situ nasal gel of midazolam prepared with Ficus carica mucilage. AAPS PharmSciTech 2010;11:1223-31.

6. Swathi S. Phytochemical screening and TLC studies of Moringa oleifera extract: their antibacterial and anti-oxidant activities Int Curr Pharm Res 2015;1:46-9.

7. Pratibha N, Saxena VS, Amit A, D'souza P, Bagchi M, Bagchi D. Antiinflammatory activities of Aller-7, a novel polyherbal formulation for allergic rhinitis. Int J Tissue Reactions 2003;26:43-51.

8. Krishnamoorthy R, Mitra AK. Prodrugs for nasal drug delivery. Adv Drug Delivery Rev 1998;29:135-46.

9. Hu G, Walls RS, Bass D, Bullock R, Grayson D, Jones M, et al. The Chinese herbal formulation biminne in management of perennial allergic rhinitis: a randomized, double-blind, placebo-controlled, 12-week clinical trial. Annals of Allergy, Asthma Immunol 2002;88:478-87.

10. Schmolka IR. Artificial skin: preparation and properties of pluronic F-127 gels for treatment of burns. J Biomed Mater Res 1972;6:571-82.

11. Sherafudeen SP, Vasantha PV. Development and evaluation of in-situ nasal gel formulations of loratadine. Res Pharm Sci 2015;10:466-76.

12. Gizurarson Sveinbjorn. Anatomical and histological factors affecting intranasal drug and vaccine delivery. Curr Drug Delivery 2012;9:566-82.

13. Bhalerao AV, Lonkar SL, Deshkar SS, Shirolkar SV, Deshpande AD. Nasal mucoadhesive in-situ gel of ondansetron hydrochloride. Indian J Pharm Sci 2009;71:711-3.

14. Miller SC, Donovan MD. Effect of poloxamer 407 gels on the miotic activity of pilocarpine nitrate in rabbits. Int J Pharm 1982;12:142-52.

15. Kumar MV, Aravindram AS, Rohitash K, Gowda DV, Parjanya K. Formulation and evaluation of in-situ gel of bromhexine hydrochloride for nasal delivery. Der Pharm Sinica 2012;3:699-707.

16. Shinde JV, Mali KR, Dias RJ, Havaldar VD, Mahajan NS. In-situ mucoadhesive nasal gels of metaclopramide hydrochloride: preformulation and formulation studies. J Pham Res 2008;1:88-96.

17. $\mathrm{Li}$ GL, Li M. Preparation and evaluation of ophthalmic thermosensitive in-situ gels of penciclovir. J Chin Pharm Sci 2007;16:90-5.

18. Schille'n K, Glatter O, Brown W. Characterization of a PEO-PPOPEO block copolymer system. Progr Colloid Polym Sci 1993;93:66-71.

19. Mainardes RM, Urban MC, Cinto PO, Chaud MV, Evangelista RC, Gremiao MP. Liposomes and micro/nanoparticles as colloidal carriers for nasal drug delivery. Curr Drug Delivery 2006;3:275-85.

20. Rana Abu-Huwaij, Rana M Obaidat, Kamal Sweidan, Yusuf AlHiari. Formulation and in vitro evaluation of xanthan gum or carbopol 934-based mucoadhesive patches, loaded with nicotine. AAPS PharmSciTech 2011;12:21-7. 